\title{
Sending Information to Interactive Receivers Playing a Generalized Prisoners' Dilemma
}

\author{
Kfir Eliaz* and Roberto Serrano ${ }^{\dagger}$
}

October 10, 2010

\begin{abstract}
Consider the problem of information disclosure for a planner who faces two agents interacting in a state-dependent multi-action prisoners' dilemma. We find conditions under which the planner can make use of his superior information by disclosing some of it to the agents, and conditions under which such information leakage is not possible. Although the problem is entirely symmetric, the planner's only way to reveal part of the information is based on creating asymmetries between the two agents by giving them different pieces of information. We also find conditions under which such partially informative equilibria are the planner's best equilibria.
\end{abstract}

JEL classification numbers: C72, D82, D83

Keywords: Information Disclosure; Generalized Prisoners' Dilemma; Uninformative Equilibria; Partially or Fully Informative Equilibria.

\section{Introduction}

There are many situations in which an informed party (e.g., a government agency, a manager, a mentor or a parent) would like to pass its knowledge on to others for whom this information is instrumental. This is especially true if given the information, there are actions that can be taken, which would benefit everyone, the party possessing the information as well as the recepients of the information. However, if the informed party cannot enforce coordination between the uninformed, then strategic externalities may lead agents who become informed to take actions that would make everyone worse off (relative to when the information was not given).

\footnotetext{
*Brown University; e-mail: Kfir_Eliaz@brown.edu; URL: http://www.econ.brown.edu/ fac/Kfir_Eliaz

${ }^{\dagger}$ Brown University and IMDEA Social Sciences Institute; e-mail: Roberto_Serrano@brown.edu; URL: http://www.econ.brown.edu/faculty/serrano
} 
Several questions arise. Should the information be disclosed and if so, how? Is it better to pass only imprecise information? Should the informed party discriminate between the agents with regards to how much information is revealed? Even if the situation described is completely symmetric among the agents, if the informed party finds he wants to reveal some information, whatever that might be, will he be telling each of them the same thing? Or will he be creating asymmetries through different pieces of information being revealed? How is the information revelation process affected by the fact that there are several receivers of the information, who are involved in a strategic interaction?

These questions are relevant in a variety of circumstances. A central bank or a government may have information about an upcoming crisis, but is concerned that if this information became known, an even bigger crisis may result if individuals rush to draw money or sell assets. The police may have information about a specific threat say, the whereabouts of a suspect or a possible bomb threat - but may be concerned that the public reaction may undermine its ability to deal with the threat (e.g., if the threat of a bomb is announced in a closed place, people might all try to rush to the same exit). A more mundane example is that of a concerned manager or a mentor who knows which tasks/projects would yield greater rewards in the long run, and which ones would yield short term gains. If he is interested in reaping the large long-term rewards, he may be concerned that if he revealed his information, competition between his underlings or advisees would lead them to choose the smaller short term gains. A related example concerns situations in which there are several ways to contribute towards a public good. If the gains and costs associated with each of these ways is known to some party, who would benefit from the public good, it may be concerned that releasing this information would lead to severe free-riding problems.

In this paper we focus on a particular environment, which to some extent illustrates all these stories, but that is motivated by the last two examples described above. In this environment, for simplicity, only two agents play a symmetric, generalized - multiaction - prisoners' dilemma, the payoffs of which depend on the state of nature. That is, the agents have finitely many actions they can coordinate on, and in each state, these actions can be ordered by the payoffs they would generate if the agents coordinated. In each state, the lowest ranked action is a dominant strategy, while the pair of highest ranked actions generate the maximal sum of payoffs. The state of nature is unknown to the agents but known to a third party, which we refer to as the planner. The planner can disclose information about the state by announcing a set of states, which include the true state. We interpret this as a requirement that only hard, verifiable evidence 
may be disclosed, and only its precision (captured by the cardinality of the reported set) can be manipulated. Also for simplicity in the analysis, we assume that the planner can only send private messages to the agents. In each state, the maximal payoff to the planner is attained when the players coordinate on the highest action.

Restricting attention throughout to pure-strategy Bayesian equilibria, we present a series of results that provide answers to the questions posed above, as a function of the degree of strength of our assumptions on the payoffs for agents and planner. While all of them are consistent with a prisoners' dilemma scenario, when one considers an arbitrary number of actions, different results obtain with different versions of the assumptions employed.

To begin with, suppose that the agents' payoffs in each state are weakly decreasing in actions (assumption A1), and that the planner prefers coordination on any action to any pair of lower actions (assumption B1). Under (A1) and (B1), Proposition 1 identifies a necessary and sufficient condition for the existence of a completely uninformative equilibrium, in which the planner does not reveal any information in any state. The condition boils down to saying that the sum of an agent's payoffs along the main diagonal of the generalized prisoners' dilemma matrix exceeds the sum of his payoffs along any other diagonal. We take this condition as the basis for the subsequent analysis, as we wish to understand whether and how much the planner can improve his ex-ante payoff with respect to this uninformative paradigm.

Our next two results (Propositions 2 and 3) are negative. The first one says that, if one restricts attention to symmetric equilibria, there is no way for the planner to improve upon the uninformative payoff. That is, under (A1) and (B1), there is no symmetric partially -or fully-informative equilibrium. Furthermore, if one strenthens (B1) to (B2), which says that the planner's payoff is increasing in actions, Proposition 3 says that there is no equilibrium - symmetric or not- that is partially or fully informative. These results point out the difficulties for the planner to make any use of his superior information in these generalized prisoners' dilemma or public good provision problems.

But, in the search of plausible circumstances to sustain equilibria with some revelation of information, we are able to offer some good news to the planner. To do this, we add a stronger assumption on agents' payoffs (A2) and we replace (B2) with a different strengthening of (B1), which we shall call (B2'). Assumption (A2) says that an agent's gains from lowering his action are always higher when the other agent is not choosing the dominant action than when he is. That is, the gains from free-riding are very small when the other agent is free-riding as well. On the other hand, (B2') 
states that, whenever there is no cooperation on the highest action, the higher the difference in actions, the higher the gains from free-riding to the free-rider with respect to the losses to the other agent. Thus, the planner, barring coordination on the efficient action, has an interest in inducing the maaximum amount of distance between the agents' actions. Under (A2) and (B2'), we show in Propositions 4 and 5 how partially informative equilibria can be constructed. Interestingly, such constructions are sensitive to the number of available actions being even or odd, which justifies the writing of two separate propositions. It is also noteworthy that the information partitions generated in equilibrium are overlapping, resembling those found in the global games literature. Finally, we show in Proposition 6 that, under (B2') and if the planner's payoff is higher under maximum separation than a suitably defined average of payoffs along the main diagonal, the equilibria constructed in Propositions 4 and 5 are actually the best equilibria for the planner.

Our paper is closely related to the cheap-talk literature that studies games between an informed "sender" and uninformed "receivers." The feature common to our model and to cheap-talk is the absence of commitment on the part of the sender. However, there are two salient distinctions. First, we make an important assumption: the planner cannot lie. That is, sooner or later he will be pressed to provide "hard facts" or evidence about the true state of nature, and punishments to lying would be prohibitive. Thus, his typical message will be of the form: "here's the set of possible states," and such a message will always contain the true state of nature as one of the possibilities. Second, we consider two strategic receivers of the message, while most of the cheap-talk literature is concerned with only one receiver. Two well-known exceptions are Farrell and Gibbons (1989) and Stein (1989). However, the first paper assumes that the payoff of each receiver is independent of the actions of the other receiver, while the second paper models the set of receivers as a single representative agent. Hence, both papers do not examine the effect of externalities across the receivers' actions on the sender's incentives.

The current paper is concerned with the effect of varying forms of information on the strategic interactions of the recipients. As such, it is also related to a recent strand of the literature, which explores the social value of information (most notably, Morris and Shin (2002) and Angeletos and Pavan (2007)). The aim of these papers is to examine the equilibrium and welfare effects of changes in the precision and form of information (private versus public) on certain classes of economies or games. A central feature of these models is that the information structure is exogenously given, whereas our main interest is in understanding what information structures would arise endogenously in 
equilibrium.

Since we model the interaction between the receivers as a generalized prisoners' dilemma, our paper is naturally related to the literature on public goods games. When there is no threshold or provision point, voluntary contribution games typically reduce to a prisoners' dilemma in which no contribution is a dominant strategy. In particular, our paper is closely related to Teoh (1997), which examines a public goods game in which the payoffs depend on a state of nature. The paper compares the equilibrium outcomes under two extreme regimes, one in which the players are perfectly informed of the state and one in which they are completely uninformed. Teoh (1997) shows that under certain conditions, the equilibrium with uninformed players is Pareto superior to the equilibrium with informed players. However, in contrast to us, Teoh (1997) does not examine what information structures would arise in equilibrium when the informed party is a player.

\section{An Illustrative Example}

To illustrate our model and some of our results, consider the following simple example. Suppose there are three equally likely states of nature, $\omega_{1}, \omega_{2}$ and $\omega_{3}$. In each state, a two-player game will be played. The true game in each state is known to the planner, but unknown to the agents. In each state, the game being played has the features of the prisoners' dilemma, i.e., a dominant action that, when chosen by both agents, leads to an inefficient outcome. However, the dominant action in question is state-dependent. Specifically, consider the following tables of payoffs, one for each state (in each state, each agent's action set consists of three actions):

\begin{tabular}{|c|c|c|c|c|c|c|c|c|c|c|c|}
\hline & $a_{1}$ & $a_{2}$ & $a_{3}$ & & $a_{1}$ & $a_{2}$ & $a_{3}$ & & $a_{1}$ & $a_{2}$ & $a_{3}$ \\
\hline$a_{1}$ & 0,0 & $2,-1$ & $5,-1$ & $a_{1}$ & 3,3 & $-1,5$ & $-1,2$ & $a_{1}$ & 1,1 & $2,-1$ & $-1,2$ \\
\hline$a_{2}$ & $-1,2$ & 1,1 & $2,-1$ & $a_{2}$ & $5,-1$ & 0,0 & $2,-1$ & $a_{2}$ & $-1,2$ & 3,3 & $-1,5$ \\
\hline$a_{3}$ & $-1,5$ & $-1,2$ & 3,3 & $a_{3}$ & $2,-1$ & $-1,2$ & 1,1 & $a_{3}$ & $2,-1$ & $5,-1$ & 0,0 \\
\hline
\end{tabular}

Thus, in state $\omega_{i}, i=1,2,3$, action $a_{i}$ is dominant for each agent. However, the efficient outcome corresponds to the action profile $\left(a_{i-1}, a_{i-1}\right)[\bmod 3]$ with payoffs $(3,3)$.

The planner, who is fully informed about the true state of nature, moves first. He communicates to each agent what states of nature are possible by sending a private message, which consists of a set of states that includes the true state. That is, the planner can control the precision of his message, but he cannot lie. The planner's 
payoff equals the sum of the agents' payoffs.

What information should the planner send the agents? Put differently, what messages does the planner send in the equilibrium with the highest expected payoff to the planner? ${ }^{1}$ There exists a pure-strategy Bayesian Nash equilibrium in which the planner discloses no information by sending each agent the message $\left\{\omega_{1}, \omega_{2}, \omega_{3}\right\}$. In this uninformative equilibrium, the agents coordinate on the same action in all states and the planner's ex-ante expected payoff is $\frac{8}{3}$.

However, the planner can do better than this by discriminating between the two agents. That is, even though the game is totally symmetric, there exists an equilibrium in which the planner earns an expected payoff of 3 by sending some information to one player and no information to the other player. Specifically, in this equilibrium there exists one player, say the row player, and one state, say $\omega_{3}$, such that in state $\omega_{3}$ the planner sends the message $\left\{\omega_{3}\right\}$ to the row player and the message $\left\{\omega_{1}, \omega_{2}, \omega_{3}\right\}$ to the column player, while in each of the other two states, the planner sends the message $\left\{\omega_{1}, \omega_{2}\right\}$ to the row player and the message $\left\{\omega_{1}, \omega_{2}, \omega_{3}\right\}$ to the column player. The row player responds to the message $\left\{\omega_{3}\right\}$ by playing the dominant action for that state, and he responds to the message $\left\{\omega_{1}, \omega_{2}\right\}$ by playing the action $a_{2}$. The column player, on the other hand, plays $a_{2}$ in response to the uninformative message.

It can be shown that this asymmetric equilibrium is the best equilibrium for the planner. In fact, there is no other equilibrium in which the planner sends some information to at least one of the players, and where at least one of the players responds to information by choosing different actions in at least two states.

In what follows we present a model that generalizes the above example. Our objective is to understand the nature of the best equilibrium for the planner, i.e., how much information does the planner disclose and does he treat the two agents symmetrically?

\section{The Basic Model}

Let $\Omega=\{1,2, \ldots, K\}$, with $K>2$, be the set of (equally likely) states of nature. ${ }^{2}$ In each state, a two-player game is played in which the payoffs are known to the planner, but unknown to the agents. In every state the set of actions available to each player is $A=\{1,2, \ldots, K\}$. Each state $\omega$ defines a linear ordering $\triangleright_{\omega}$ over the actions, such

\footnotetext{
${ }^{1}$ The equilibria that we describe here do not rely on allowing any out-of-equilibrium beliefs. The particular restriction that we imposed will be described in Section 4.

${ }^{2}$ We shall discuss the case of $K=2$ in our concluding section. The uniform distribution assumption is made for convenience; obviously, the statemens and conditions would need to be adjusted accordingly were one to use a different distribution.
} 
that

$$
\begin{aligned}
& 1 \triangleleft_{1} 2 \triangleleft_{1} \ldots \triangleleft_{1} K-1 \triangleleft_{1} K \\
& 2 \triangleleft_{2} 3 \triangleleft_{2} \ldots \triangleleft_{2} K \triangleleft_{2} 1 \\
& \vdots \\
& K \triangleleft_{K} 1 \triangleleft_{K} \ldots \triangleleft_{K} K-2 \triangleleft_{K} K-1
\end{aligned}
$$

If $a \triangleleft_{\omega} b$ for some actions $a, b \in A$, we say that action " $b$ is higher than $a$ at $\omega$ ". We interpret $\triangleleft_{\omega}$ as a state-dependent ranking of the "intensity" or "strength" of the actions. A "high" action in one state may be considered a "low" action in another state. The player's payoff would therefore depend on the "intensity/strength" of the players' actions. I.e., the outcome of the action pair $(1,2)$ in state 1 is equivalent to the outcome of the action pair $(2,3)$ in state 2 . Furthermore, actions are ex-ante symmetric, in the sense that for any given action there exists a state in which it is the highest, another state in which it is the lowest, and states in which it occupies any intermediate position.

To express all this formally, for any $a \in A$ and $\omega \in \Omega$, define $s(a, \omega)$ as the number of elements in $A$ that are ranked below $a$ according to $\triangleleft_{\omega}$. I.e.,

$$
s(a, \omega)=\#\left\{b \in A: b \triangleleft_{\omega} a\right\}
$$

For any $a \in A$ and $\omega \in \Omega$, we define

$$
x(a, \omega) \equiv\left\{a^{*} \in A: s(a, \omega)=s\left(a^{*}, 1\right)\right\}
$$

Note that for every $\omega \in \Omega, \omega$ is the lowest ranked action according to $\triangleleft_{\omega}$. Since the "intensity/strength" of actions varies with the state, we interpret $x(a, \omega)$ as the "strength/intensity" of $a$ at $\omega$, normalized to state 1 terms. Player $i$ 's payoff in state $\omega$ from the action pair $\left(a_{1}, a_{2}\right)$ is denoted $u_{i}\left(a_{1}, a_{2} \mid \omega\right)$. Given our normalization to actions in state 1 , denote $u_{i}\left(a_{1}, a_{2} \mid 1\right)$ by $u_{i}\left(a_{1}, a_{2}\right)$, and we assume that for $i=1,2$ and for all $a_{1}, a_{2}$ and $\omega, u_{i}\left(a_{1}, a_{2} \mid \omega\right)=u_{i}\left[x\left(a_{1}, \omega\right), x\left(a_{2}, \omega\right)\right]$. We assume the game is symmetric: for any $x, y \in\{1, \ldots, K\}, u_{i}(x, y)=u_{j}(y, x)$. Hence, to simplify the exposition we write $u(x, y)$ to denote the payoff to a player who chooses $x$, while the other player chooses $y$.

The payoff to the planner is given by the sum of the players' payoffs. That is, the planner who knows that the true state is $\omega$, when the agents choose actions $a_{1}$ and $a_{2}$ 
receives a payoff of $V\left(x\left(a_{1}, \omega\right), x\left(a_{2}, \omega\right)\right)$ :

$$
V\left(x\left(a_{1}, \omega\right), x\left(a_{2}, \omega\right)\right)=u\left[x\left(a_{1}, \omega\right), x\left(a_{2}, \omega\right)\right]+u\left[x\left(a_{2}, \omega\right), x\left(a_{1}, \omega\right)\right]
$$

With the assumptions made so far, $V$ is symmetric so that $V(x, y)=V(y, x)$.

The game played in each state is interpreted as a generalized multi-action Prisoners' Dilemma. To capture this interpretation we make a number of assumptions on payoffs. We group them in assumptions on the players' payoffs (denoted by the letter A) and on the planner's payoffs (denoted by the letter B):

The first assumption on players' payoffs one could start with is that the lowest action in each state is strictly dominant: for any $x>1$ and for any $y$,

$$
u(1, y)>u(x, y)
$$

However, because of the multi-action framework, this will not suffice to obtain most of our results. Hence, we strenghthen it as follows:

(A1) Each player has a weak incentive to lower his action: for all $x>x^{\prime} \geq 1$ and $y$,

$$
u\left(x^{\prime}, y\right) \geq u(x, y)
$$

with a strict inequality for $x^{\prime}=1$.

Assumption (A1) captures the basic intuition in a generalized prisoners' dilemma logic, that each player has a preference for lowering his action/effort, ceteris paribus. For some of our last results, we shall add another assumption in addition to (A1), which we introduce as follows.

First, consider the change in an agent's payoff when he lowers his action to the dominant one, in a state where his opponent chooses any action other than the dominant:For any $k>1$,

$$
\Delta_{k}(a) \equiv u(1, k)-u(a, k)
$$

Consider next the change in the player's payoff when he lowers his action from the highest action in a state where his opponent chooses the dominant action:

$$
\Delta_{1}(a) \equiv u(a, 1)-u(K, 1)
$$

By (A1), while $\Delta_{k}(a)$ is increasing in $a, \Delta_{1}(a)$ is decreasing. Consider the following condition, 
(A2) For any $k>1, \min _{a>1} \Delta_{k}(a)>\max _{a} \Delta_{1}(a)$ or given (A1): $\Delta_{k}(2)>\Delta_{1}(1)$.

To interpret condition (A2), one should think of the gains associated with lowering one's action. The assumption says that any such gain when the opponent is not playing the dominant action exceeds any such gain when he is. The individual gains from exerting less effort are very small if the other agent is already exerting the smallest amount of effort.

Next, we move to assumptions on the planner's payoffs. Two basic features, consistent with a prisoners' dilemma scenario, that we would like to capture are the following:

On the one hand, coordination on the lowest action is the worst outcome for the planner: for any $x \geq 1, y \geq 1$ with at least one strict inequality,

$$
V(1,1)<V(x, y)
$$

On the other hand, coordination on the highest action is the best outcome for the planner: for any $x \leq K, y \leq K$ with at least one strict inequality,

$$
V(K, K)>V(x, y)
$$

As before, we shall need to strenghthen these basic features in order to obtain results in our multi-action model. That is, to capture a sense in which coordination on an intermediate action - i.e., coordination on some $a<K$ - is also beneficial for the planner, we begin by considering the following:

(B1). The planner prefers coordination on any action $1<x \leq K$ to any pair of lower actions: for any $y \leq z \leq x$,

$$
V(x, x) \geq V(y, z)
$$

A stronger version of (B1), which we shall use in one of the results, offers a complete conflict of interest between planner and players. That is, while (A1) stipulates that players' payoffs are decreasing in the player's action, assumption (B2) poses that the planner's payoffs are increasing in actions:

(B2) the planner's payoff increases with the players' actions: if $x>x^{\prime}$, then for every $y, V(x, y)>V\left(x^{\prime}, y\right)$.

Assumptions (A1) and (B2) may be interpreted as a public good problem in which the agents face uncertainty regarding the cost of investing in the public good. Assumption (A1) can be viewed as saying that the more an action contributes to the optimal public good, the more costly it is, so that agents have a strict incentive to 
free-ride. Assumption (B2) means that the planner is interested in the highest amount of contribution.

In contrast to Assumption (B2), Assumption (B2'), presented next, implies that the planner's payoff increases when players' difference in actions rises. Namely, we assume that if for some reason the planner knows that the agents will not cooperate in the highest action, then he prefers that one agent undercuts the other with the highest margin (note that while this is a departure from (B2), it is still compatible with (B1)). As will be seen, whether one assumes (B2) or (B2'), the information disclosure happening in equilibrium will be significantly different. Assumption (B2') follows:

(B2') For any $x<y<z$,

$$
V(x, z)>\max \{V(x, y), V(y, z), V(y, y)\}
$$

Note in particular how the example in Section 2 satisfies Assumptions (A1), (A2) (this is satisfied with a weak inequality that could be made strict without altering the conclusions), (B1) and (B2'). However, it violates Assumption (B2).

\section{Analysis and Results}

The planner's information disclosure strategy can be described as follows: in each state $\omega$ he sends a pair of private messages $\left(m_{1}, m_{2}\right)$, where $\omega \in m_{i} \subseteq \Omega$. That is, the planner announces a list of possible states, but one of the states he announces must be the true state. The idea is that the planner cannot lie, perhaps because there are prohibitive penalties to doing so when the state is eventually revealed.

We focus on pure-strategy Bayesian Nash equilibria (BNE) using the following restrictions on out-of-equilibrium beliefs:

$\left(\beta_{1}\right)$ Consistent with the idea of independent trembles, a player who finds himself off the equilibrium path believes that only he received an out-of-equilibrium message (i.e., he believes the other player received a message according to equilibrium).

$\left(\beta_{2}\right)$ Consistent with the logic of some refinements (e.g., the intuitive criterion), a player assigns a positive probability to the event that a planner of type $\omega$ deviated from equilibrium only if this planner type has an incentive to deviate in this state.

We shall use the above restrictions for the construction of the equilibria in Propositions 1, 4 and 5. All other results do not rely on these restrictions. 
We proceed to our results. We begin by stating an auxiliary lemma:

Lemma 1 If (A1) holds, then any BNE has the following property. If an agent believes, both on or off the equilibrium path, that a set of states $S$ is possible, he must choose an action, which is dominant in one of these states.

Proof. Consider a BNE, a player, say 1, and a planner type $\omega$. Let $S \subseteq \Omega$ be the message that the planner sends to player 1 in state $\omega$. If $S=\Omega$ then the Lemma is trivially true. Suppose therefore that $S \subset \Omega$ and player 1 responds by choosing an action $a$, which is not dominant in any of the states in $S$. Then, there exists a state $\omega^{\prime} \in S$ satisfying $x\left(a, \omega^{\prime}\right)<x\left(a, \omega^{\prime \prime}\right)$ for all $\omega^{\prime \prime} \in S$. Since $a$ is not dominant in $\omega^{\prime}$, $x\left(a, \omega^{\prime}\right)>1$. By our assumption on how the ranking of actions changes across states and by our choice of $\omega^{\prime}$, any action $b$ with $x\left(b, \omega^{\prime}\right)<x\left(a, \omega^{\prime}\right)$ satisfies $x\left(b, \omega^{\prime \prime}\right)<x\left(a, \omega^{\prime \prime}\right)$ for all $\omega^{\prime \prime} \in S$. Let $b^{*}$ be the dominant action in state $\omega^{\prime}$, i.e., $x\left(b^{*}, \omega^{\prime}\right)=1$.By (A1), if player 1 deviated to $b^{*}$ his expected payoff would strictly increase as his payoff would increase in every state in $S$, a contradiction.

\subsection{Completely Uninformative Equilibria}

We can now present our first main result, which states a necessary and sufficient condition for the existence of a completely uninformative equilibrium. First, we present the following definition:

We shall say that a BNE is symmetric when: (i) for all $\omega$, the type $\omega$ planner sends the same message $S(\omega)$ to player 1 and player 2, and (ii) both players 1 and 2 use the same strategy $a(S(\omega))$ for all messages $S(\omega)$.

Proposition 1 Assume (A1) and (B1) hold. Then there exists a symmetric BNE in which the planner sends the uninformative message $\Omega$ in every state if and only if

$$
\sum_{k=1}^{K} u(k, k) \geq \sum_{k=1}^{K} u\left((k+\delta)_{\bmod K}, k\right)
$$

for any integer $\delta$.

Remark: We can term this condition "dominant main diagonal." Indeed, if one writes the matrix of payoffs for player 1 and one copies the first $K-1$ rows below that matrix, 
the condition states that the sum of elements along the main diagonal exceeds the sum of all diagonals (of the expanded matrix) placed below it. Given that it is necessary for the existence of the uninformative equilibrium, this is the condition we shall focus on in the general model, if we wish to study when a planner can improve his payoff by revealing some information.

Proof of Proposition 1. Consider the following profile of strategies. Every planner type sends the message $\Omega$ to each of the players, and each player responds to $\Omega$ by choosing the same action. Assume, without loss of generality, that this action is $K$. For any message $S \neq \Omega$, call $\omega \in S$ the state such that $\omega^{\prime}<\omega$ for all $\omega^{\prime} \in S \backslash\{\omega\}$, let a player assign probability 1 to state $\omega$ and choose the dominant action in state $\omega$. Note that this covers the case of singleton messages and also the case of any message containing state $K$, to which the player would respond by continuing to choose action $K$. We now show that this strategy profile is a BNE under inequality (2).

Consider each player first. By (2), no player has an incentive to play $a<K$ in response to the message $\Omega$, given that the other player responds with $K$. In addition, in every state $\omega>1$ the planner has an incentive to try and change the players' actions. Hence, in particular, the players' out-of-equilibrium beliefs are consistent with $\left(\beta_{1}\right)-\left(\beta_{2}\right)$.

Consider the planner next. In each state $\omega$ the proposed strategy profile generates a payoff of $V(1+K-\omega, 1+K-\omega)$. The planner has no incentive to send both players the same message because they will respond to it by coordinating on a lower action in that state: indeed, if the type $\omega$ planner sent the message $S \neq \Omega$ to both players with $k^{\prime}$ being the maximal state in $S$, the planner's resulting payoff would be $V\left(1+k^{\prime}-\omega, 1+k^{\prime}-\omega\right) \leq V(1+K-\omega, 1+K-\omega)$ by $(\mathrm{B} 1)$.

Suppose now the planner deviates by sending different messages to either player: $S_{1}$ with maximal element $k_{1}$ to player 1 , and $S_{2}$ with maximal element $k_{2}$ to player 2 . Given the players' beliefs, the type $\omega$ planner's payoff would be $V\left(1+k_{1}-\omega, 1+k_{2}-\omega\right)$. Note that, since the planner cannot lie, $k_{1} \geq \omega$ and $k_{2} \geq \omega$. Hence, by (B1), the planner weakly prefers that the players coordinate on $1+K-\omega$.

Conversely, if there exists a symmetric uninformative BNE, then there exists some action $a$ that both players choose in every state. Since no player has an incentive to deviate, inequality (2) must hold.

If the uninformative equilibrium exists, it generates the following ex-ante expected 
payoff to the planner:

$$
\bar{V} \equiv \frac{1}{K} \sum_{k=1}^{K} V(k, k)
$$

We shall call this "the uninformative payoff".

\subsection{When Partially Informative Equilibria Are Impossible}

Next, we turn to the investigation of equilibria in which the planner gives out some information. A BNE is said to be partially informative if for some player, at least two types choose different actions in equilibrium. The next result offers an impossibility, i.e., for the planner to give out some information, asymmetries in the equilibrium will be required.

Proposition 2 Assume (A1) and (B1) hold. Then there is no symmetric BNE with partial information.

Proof of Proposition 2. Assume, by contradiction, that there exists a symmetric BNE with this property. Let $a$ be an action that is played in equilibrium. By Lemma 1 , there must be some state $\omega$ such that $a$ is played by both players in $\omega$ and $a$ is dominant in $\omega$. Suppose $b \neq a$ is the players' response to the uninformative message $\Omega$. Then by (B1), a type $\omega$ planner can profitably deviate by sending both players the message $\Omega$. It follows that $a$ must be the players' response to the message $\Omega$.

By assumption, the players in equilibrium also coordinate on another action $a^{\prime} \neq a$. Again, by Lemma 1, both players coordinate on $a^{\prime}$ in a state $\omega^{\prime}$ in which $a^{\prime}$ is the dominant action. But then by (B1), a type $\omega^{\prime}$ can profitably deviate by sending both players the message $\Omega$. Since it cannot be the case that the players respond to $\Omega$ with both $a$ and $a^{\prime}$, it follows that there cannot be a BNE with the stated properties.

Proposition 2 implies the following:

Corollary 1 Assume (A1) and (B1) hold. There exists no BNE with full information.

Proof. This follows since the full information outcome would obtain from a symmetric profile, in which each player is told the true state by the planner in each state $\omega$. 
We proceed now to explore the consequence of our first strenghthening of the assumption on the planners's payoff, i.e., Assumption (B2):

Proposition 3 Assume (A1) and (B2) hold. There is no BNE with partial information.

Proof of Proposition 3. Suppose there exists a BNE with partial information. By assumption, there exist two states in which one of the players, say player 1, chooses different actions, say $a$ and $a^{\prime}$, in equilibrium. By Lemma 1 there exist a pair of states, call them $\left(\omega, \omega^{\prime}\right)$, such that $a$ is dominant in $\omega$ and $a^{\prime}$ is dominant in $\omega^{\prime}$. Hence, $x(b, \omega)>x(a, \omega)$ for all $b \neq a$ and $x\left(b^{\prime}, \omega^{\prime}\right)>x\left(a^{\prime}, \omega^{\prime}\right)$ for all $b^{\prime} \neq a^{\prime}$.

Let $b^{*}$ denote player 1's equilibrium response to the uninformative message $\Omega$. If $b^{*} \neq a$, then (B2) implies that in state $\omega$ the planner can profit by sending the message $\Omega$ only to player 1 , regardless of player 2 's action in that state. If $b^{*}=a$, then again by (B2), in state $\omega^{\prime}$ the planner can profit by sending $\Omega$ only to player 1 . This means that at least in one state, the planner has a profitable deviation, a contradiction.

Proposition 3 then implies that in the types of public good problems fitting Assumptions (A1) and (B2), there is no pure-strategy equilibrium that is better for the planner than the uninformative equilibrium.

\subsection{When Partially Informative Equilibria Are Possible}

We explore next some of the circumstances under which it is in the interest of the planner to disclose some information in equilibrium. In doing so, consider instead a different strenghthening of (B1): we shall assume (B2') instead of (B2). We shall also strenghthen (A1) into (A2). We note that these are sufficient conditions, but not necessary (we know we can prove the next results under weaker conditions).

Recall that the planner is assumed to care about the sum of the agents' payoffs. Condition (A2) does not relate the gain of agent $i$ from undercutting $j$ to the loss of $j$ from being undercut by $i$. It just states that the shirking gains to "doing nothing" are uniformly higher when the other colleague is exerting high effort than when he is exerting the lowest - perhaps through the positive externalities that the colleague's high effort brings to the agent. Condition (A2) therefore is not concerned with the planner's payoff. In other words, conditional on the event that the agents do not cooperate, it does not capture the effect of undercutting on "social surplus". Such a 
concern is captured in (B2'), in which we assume that if for some reason the planner knows that the agents will not cooperate efficiently, then he prefers that one agent undercuts the other with the highest margin. Extreme differences are preferred to average differences in efforts, a strong form of "non-convexity."

The following lemma gives a hint about a basic feature of the equilibria we are seeking:

Lemma 2 Assume (B2'). Any BNE with partial information has the property that in every state where players mis-coordinate, some player chooses the dominant action.

Proof.Let $\omega$ be a state in which the two agents choose different actions $a$ and $b$ with $x(a, \omega)<x(b, \omega)$. We argue by contradiction. Then $x(a, \omega)>1$. By (B2'), the planner can profitably deviate by sending the message $\{\omega\}$ to the agent choosing $a$, a contradiction.

We turn to our results that construct partially informative equilibria.

Proposition 4 Assume (A2) and (B2') hold. Let $K$ be even. Then there exists a partially informative equilibrium, which has the following structure. The planner gives out information so as to create the following partitions: $\{\{1,2\},\{3,4\}, \ldots,\{K-1, K\}\}$ for player 1, and $\{\{2,3\},\{4,5\}, \ldots,\{K, 1\}\}$ for player 2. Then, on the equilibrium path, players 1 and 2 choose action $k$ following the information $\{k, k+1\}$ (note that this implies that, in each state, one player chooses the dominant action and the other chooses the highest action).

Proof of Proposition 4. Suppose $K$ is even. The planner's strategy is implicit in the statement of the proposition. As for the players' strategies, they are as follows. On the equilibrium path, they are also described in the statement. Off the equilibrium path, given any singleton message $\{k\}$, they choose the dominant action in that state. For any other message, player 1 assigns probability one to the lowest odd state, while player 2 assigns probability one to the highest even state, and their response is to play the corresponding dominant action. If a message contains only even states, player 1 assigns probability one to the lowest even state and chooses the dominant action for that state. Similarly, if a message contains only odd states, then player 2 chooses the dominant action for the highest odd state. 
To check that this is a BNE of this game, we define a player's type space to be the set of messages he receives in the proposed equilibrium. The planner's type is naturally defined to be the state of nature. We begin by verifying that no player type has any incentive to deviate. Consider type $\{k, k+1\}$ of player 1 . By playing $a=k$, this type obtains an expected payoff of

$$
\frac{1}{2} u(1, K)+\frac{1}{2} u(K, 1)=\frac{1}{2} V(1, K) .
$$

Playing any $a^{\prime}>1$ would yield an expected payoff of

$$
\frac{1}{2} u\left(a^{\prime}, K\right)+\frac{1}{2} u\left(a^{\prime}-1,1\right) .
$$

By (A2) applied to $k=K, \Delta_{K}\left(a^{\prime}\right)>\Delta_{1}\left(a^{\prime}-1\right)$, i.e., $u(1, K)-u\left(a^{\prime}, K\right)>u\left(a^{\prime}-1,1\right)-$ $u(K, 1)$, which implies that no deviation to taking action $a^{\prime}$ is profitable. Of course, the argument for player 2 is identical.

Consider now the planner, who receives a payoff of $V(1, K)$. From (B2'), it follows that the only way a planner of type $k$ can increase his payoffs is to induce the players to coordinate on an action $a \neq k$. With the strategies written, we show this is impossible. Indeed, suppose first $k$ is odd. To induce the players to coordinate on an action $a$, the message that each player receives must contain both $k$ and $a$. If the message to player 2 also contains an even action, then players would not coordinate as player 2 would choose an even action while player 1 would choose an odd action. This implies that $a$ must be odd. This also means that the message sent to player 2 must contain only odd actions. Since player 1 would choose the lowest odd action in his message, while player 2 would choose the highest odd action, and since the message to each player must contain both $k$ and $a$, the two players would not be able to coordinate on $a$. By a similar argument, the planner cannot induce the players to coordinate when $k$ is even. It follows that in no state can the planner send an admissable message (i.e., a message containing that state) that will induce both players to coordinate on an action, unless this is the dominant action in that state. Hence, no planner type has any incentive to deviate.

We next address the case of odd $K$ :

Proposition 5 Assume (A2) and (B2') hold. Let $K$ be odd. There exists a partially informative equilibrium, which has the following structure. The planner gives out information so as to create the following partitions: $\{\{1,2\},\{3,4\}, \ldots,\{K-2, K-1\},\{K\}\}$ 
for player 1, and $\{\{2,3\},\{4,5\}, \ldots,\{K-1, K, 1\}\}$ for player 2. Then, on the equilibrium path, players 1 and 2 choose action $k$ following the announcements $\{k\},\{k, k+1\}$ or $\{k, k+1, k+2\}$ (note that this implies that in all states but one a player chooses the dominant action and the other chooses the highest action. In state 1, in contrast, one player chooses the dominant action and the other chooses the second highest action).

Proof of Proposition 5. Suppose $K$ is odd. The planner's strategy is implicit in the statement of the proposition. As for the players' strategies, they are as follows. On the equilibrium path, they are also described in the statement. For any other message, player 1 assigns probability one to the lowest odd state, while player 2 assigns probability one to the highest even state, and their response is to play the corresponding dominant action. If a message contains only even states, player 1 assigns probability one to the lowest even state and chooses the dominant action for that state. Similarly, if a message contains only odd states, then player 2 chooses the dominant action for the highest odd state, except for the case where the message contains both the lowest and the highest states, in which case player 2 chooses the dominant action for the second highest state.

To check that these strategies constitute a BNE of this game, we define a player's type space to be the set of messages he receives in the proposed equilibrium. The planner's type is naturally defined to be the state of nature. We begin by verifying that no player type has any incentive to deviate. First, type $\{K\}$ of player 1 clearly has no incentive to deviate as he chooses a dominant action. Next, consider type $\{1,2\}$ of player 1 . By playing $a=1$ type $\{1,2\}$ obtains an expected payoff of

$$
\frac{1}{2} u(1, K-1)+\frac{1}{2} u(K, 1)
$$

while playing any $a>1$ would yield an expected payoff of

$$
\frac{1}{2} u(a-1, K-1)+\frac{1}{2} u(a-2,1)
$$

By (A2) applied to $k=K-1$,

$$
u(1, K-1)-u(a-1, K-1)>u(a-2,1)-u(K, 1)
$$

Finally, consider a type $\{k, k+1\}$ of player 1 . By playing $a=k$, this type obtains an expected payoff of

$$
\frac{1}{2} u(1, K)+\frac{1}{2} u(K, 1)
$$


Playing any $a^{\prime} \neq k$ would yield an expected payoff of

$$
\frac{1}{2} u\left(a^{\prime}, K\right)+\frac{1}{2} u\left(a^{\prime}-1,1\right) .
$$

By (A2) applied to $k=K, u(1, K)-u\left(a^{\prime}, K\right)>u\left(a^{\prime}-1,1\right)-u(K, 1)$, which implies that no deviation to taking action $a^{\prime}$ is profitable.

To see that player 2 has no incentive to deviate, note first that any type $\{k, k+1\}$ of player 2 has no incentive to deviate for the same reason that a type $\{k, k+1\}$ of player 1 has no incentive to deviate. Consider then type $\{K-1, K, 1\}$ of player 2 . By playing $K-1$, this type obtains an expected payoff of

$$
\frac{1}{3} u(1, K)+\frac{1}{3} u(K, 1)+\frac{1}{3} u(K-1,1)
$$

Playing any $a \neq K-1$ would yield an expected payoff of

$$
\frac{1}{3} u(a, K)+\frac{1}{3} u(a-1,1)+\frac{1}{3} u(a-2,1)
$$

By (A2) applied to $k=K$,

$$
u(1, K)-u(a, K)>[u(a-1,1)-u(K, 1)]+[u(a-2,1)-u(K-1,1)]
$$

for all $a \neq K-1$.

Consider now the planner, who receives a payoff of $V(1, K)$ in every state except for state 1 , where he receives a payoff of $V(1, K-1)$. In every state other than 1 , the planner can increase his payoff by inducing the players to coordinate on a high enough action $a$. In state 1 , the planner can also profit by inducing maximum separation (one player choosing the lowest and the other the highest action).

Consider first planners of type $\omega>1$. The reason this planner cannot achieve coordination follows from essentially the same arguments given in the proof of Proposition 4. The only modification that needs to be made is when player 2 receives a message with only odd states, and which contains states 1 and $K$. If the message contains at least one other state, then the two players would mis-coordinate. If the message is $\{1, K\}$, then both players would choose action 1 . However, for the planner types we are currently concerned with, this message can only be sent in state $K$, and then, by (B2'), the planner prefers the equilibrium actions than coordination on action 1: $V(1, K)>V(2,2)$.

Finally, consider the type 1 planner. Note that player 1 will always choose action 
1, for any message sent by the planner. Getting the two players to coordinate is impossible, unless player 2 is sent the message $\{1, K\}$, but then coordination is on action 1, which is worse for the planner than the equilibrium. And getting player 2 to choose action $K$ to induce maximum separation is also impossible, given the strategies written.

It follows that the planner has no profitable deviation.

Propositions 4 and 5 show that under certain conditions there exist partially informative equilibria, which are asymmetric even though the underlying game in each state is completely symmetric. In addition, the informational structures induced in these equilibria are similar in nature to the typical information structures in global games.

\subsection{Social Evaluation of the Partially Informative Equilibria}

A natural question that arises is under what conditions would these equilibria maximize the ex-ante expected payoff to the planner. One could rephrase this question as follows: under what conditions would the informational structures that are typically assumed in global games could be explained as being induced by an informed planner in the equilibrium that is best for him?

To address this question we introduce the following notation. For any $M \subseteq$ $\{2, \ldots, K-1\}$ let

$$
W(M)=\frac{1}{|M|+2}\left[\sum_{m \in M} V(m, m)+V(1,1)+V(K, K)\right] .
$$

Proposition 6 Assume (B2') holds. If $K$ is even and $V(1, K) \geq W(M)$ for all $M \subseteq$ $\{2, \ldots, K-1\}$, then there is no BNE with a higher ex-ante payoff to the planner than the equilibrium described in Proposition 4. If $K$ is odd and $V(1, K-1) \geq W(M)$ for all $M \subseteq\{2, \ldots, K-1\}$, then there is no BNE with a higher ex-ante payoff to the planner than the equilibrium described in Proposition 5.

Proof of Proposition 6. Assume $K$ is even and suppose, by contradiction, that there is an equilibrium with a higher ex-ante payoff to the planner. In that equilibrium, partition the set of states into two categories, those states in which players mis-coordinate and those in which they coordinate. In the equilibrium of Proposition 
4, the planner obtains a payoff of $V(1, K)$ in each state. Consider some state in the mis-coordination category and let $V(x, y)$ be the payoff to the planner in that state, where $x \geq 1$ and $y \leq K$. By (B2'), $V(1, K) \geq V(x, y)$. Let $M$ be the set of states in the coordination category. Note that for every state in which the players coordinate on some action $x>1$, there exists a state in which they coordinate on $x=1$. To see why, assume without loss of generality, that in state 1 the players play action $k>1$. Obviously, the planner in state 1 must be sending a message to each player that contains both state 1 and state $k$, and hence, for each state in which the planner is receiving a payoff $V(k, k)$, there exists another state in which the planner is receiving $V(1,1)$. Furthermore, if there exists a pair of states in which the players coordinate on different actions, then there exists a pair of other states where the players play the dominant action for those states. By assumption, $V(1, K) \geq W(M)$. Since each state is equally likely, the ex-ante payoff to the planner in the hypothesized equilibrium cannot be higher than $V(1, K)$. Essentially the same argument applies for the case of odd $K$.

\section{Concluding Remarks}

We have studied the problem of information disclosure for a planner who faces two agents interacting in a state-dependent generalized prisoners' dilemma. We have found conditions under which the planner can make use of his superior information by giving some of it out to the agents, and conditions under which such information leakage is not possible. We remark that, although the problem is entirely symmetric, the planner's only way to reveal part of the information is based on creating asymmetries between the two agents by giving them different pieces of information. We have also found conditions under which such partially informative equilibria are the best equilibria from the planner's point of view.

In our study, we have assumed that the number of actions -and states- was at least three. The two-action case, which depicts the standard prisonners' dilemma, yields the following. Under the "dominant main diagonal" condition 2 in Proposition 1 , the completely uninformative equilibrium exists, but no other equilibrium can be found to dominate it. If this condition is violated, akin under $K=2$ to assuming (B2'), the partially informative equilibrium of Proposition 4 is found. Unfortunately, its performance cannot be compared to the completely uninformative paradigm, which in this case is not an equilibrium. Thus, it appears that the two-action case gives one a vastly simplified picture of the problem: information disclosure is possible if and only 
if the sum of the off-diagonal payoffs in the prisonners' dilemma dominate the sum of payoffs on the main diagonal. In contrast, the multi-action case we study allows condition 2 and assumption (B2') to hold simultaneously and provides a more complex set of results.

\section{References}

[1] Angeletos, G.-M. and A. Pavan (2007): "Efficient Use of Information and Social Value of Information," Econometrica, 75, 1103-1142.

[2] Farrell, J. and R. Gibbons (1989): "Cheap Talk with Two Audiences," American Economic Review, 79, 1214-1223.

[3] Morris, S. and H.S. Shin (2002): "The Social Value of Information," American Economic Review, 92, 1521-1534.

[4] Stein, J. C. (1989): "Cheap Talk and the Fed: A Theory of Imprecise Policy Announcements," American Economic Review, 79, 32-42.

[5] Teoh, S. H. (1997): "Information Disclosure and Voluntary Contributions to Public Goods," RAND Journal of Economics, 28, 385-406. 